Mirai. Estudios Japoneses

ISSN-e: 2531-145X

http://dx.doi.org/10.5209/MIRA.60492

\title{
Giovanni Cola (Joao Nicolao). Un hombre del renacimiento italiano trasplantado a Japón ${ }^{1}$
}

\author{
S.J. Fernando $\mathrm{G}^{\mathrm{a}}$ Gutiérrez
}

Fechas

Resumen. Los contactos históricos entre Oriente y Occidente han sido siempre enriquecedores para ambas partes. Así ocurrió también en la historia del arte: las influencias mutuas fueron el origen de nuevas corrientes estéticas. Giovanni Cola, un jesuita con una enorme capacidad de enculturación, supo penetrar en el mundo cultural del Lejano Oriente, y a la vez fue portador de los valores artísticos de Occidente, sobre todo para Japón.

Palabras clave: Giovanni Cola; Joao Nicolao; pintor; jesuita; Japón.

\section{[en] Giovanni Cola (Joao Nicolao). A Renaissance Italian in Japan}

\begin{abstract}
Mutual contacts between East and West along the history have always been a cause of enrichment for both parts. The same enrichment has happened in the history of art: mutual influences are in the origin of new artistic streams. Giovanni Cola, a Jesuit of tremendous capacity for inculturation, was able to penetrate in the culture of the Far East and, at the same time, he afforded the artistic values of western art, especially to Japan.
\end{abstract}

Keywords: Giovanni Cola; Joao Nicolao; painter; Jesuit; Japan.

Cómo citar: Fernando Ga Gutiérrez, S.J. (2018). Giovanni Cola (Joao Nicolao). Un hombre del renacimiento italiano trasplantado a Japón, en Mirai. Estudios Japoneses 2(2018), 3-19.

Los contactos entre Oriente y Occidente a través de la historia han sido siempre una fuente de enriquecimiento para las dos partes. Cuando todavía no existían los medios de comunicación, la distancia hacía que las distintas culturas estuvieran muy alejadas. Por eso, las influencias mutuas también eran escasas y, sin embargo, cuando las circunstancias proporcionaban algún contacto, los resultados eran siempre enriquecedores. Así ocurrió también en la historia del arte: las influencias mutuas de Oriente y Occidente han dado como resultado nuevas corrientes estéticas, con aspectos de

1 La publicación de este artículo es una reedición del aparecido en la revista Temas de estética y arte, ISSN 02146258, No 25, 2011, pp. 96-124, publicada por la Real Academia de Santa Isabel de Hungría de Sevilla. Quiere ser un sencillo homenaje a este primer investigador de la Historia del Arte Japonés en España. Por tal motivo, no se ha adaptado a las normas de Mirai. Estudios Japoneses y se han respetado los criterios de la antigua publicación en la que apareció. Este trabajo ha sido elegido por estar en consonancia con su persona, la imagen de un misionero y su relación con el arte en Japón. Nuestro sincero agradecimiento a la Real Academia de Bellas Artes Santa Isabel de Hungría por su gentileza. 
originalidad para ambas partes ${ }^{2}$. Estos contactos históricos entre Oriente y Occidente fueron el origen de estilos nuevos, que surgían al conocerse mutuamente las culturas que, por circunstancias históricas, se habían desarrollado independientemente. La figura de Giovanni Cola, con su enorme capacidad de enculturación, supo penetrar en el mundo cultural del Lejano Oriente, a la vez que fue portador de los valores artísticos de Occidente, de donde provenía, y dio origen a escuelas de arte enteramente originales, en que se daba la unión de valores hasta entonces desconocidos.

\section{Giovanni Cola (1560-1626)}

Japón está descrito en el Libro de las maravillas (Il Milione) de Marco Polo (12541324), que él mismo había titulado El Libro de Marco Polo. Es una descripción altamente original e imaginativa, que seguramente recibió de algún comerciante chino que se había dejado llevar de su imaginación. En uno de los capítulos del libro de sus viajes, Marco Polo describe a Japón de esta manera:

Zipangu (Japón) es una isla del océano oriental a una distancia de mil quinientas millas de la tierra firme, o costa del Mangi (China)... El rey de la isla tiene un gran palacio techado con finísimo oro, tal como nuestras iglesias lo están con pizarra. Todas las ventanas están decoradas con oro; los pisos de los salones y de muchas cámaras están pavimentados con planchas de oro, cada una de ellas con sus buenos dos dedos de espesor. Se encuentran allí perlas de gran abundancia, de un color rosado y de forma redonda. También se encuentran allí gran número de piedras preciosas... ${ }^{3}$

Esta descripción de Japón resulta curiosa: nunca ha habido en toda la historia de Japón palacios recubiertos de oro, ni existen en aquel país las piedras preciosas que dice hay en gran abundancia. Lo único verosímil es la abundancia de perlas, que se apreciaban allí desde los tiempos más antiguos. Sin duda recibió Marco Polo esta descripción de las islas japonesas de algún comerciante de una imaginación inventiva.

Japón tuvo su primer contacto directo con el mundo occidental cuando una nave portuguesa arribó a la isla de Tanegashima, ante la costa de Kyushu, en $1542^{4}$. La verdadera influencia del arte occidental en Japón empezó más tarde con la llegada de los primeros misioneros cristianos: fue San Francisco Javier el portador de los primeros objetos de arte europeo, que produjo un impacto cierto en Japón. Shimazu Takahisa (1414-1571), señor feudal de la región de Shimazu, trató de conseguir datos sobre Europa e India a través de Anjiro, el intérprete japonés que había llevado Javier desde Goa. Anjiro había recibido su formación religiosa de Javier, y al mismo tiempo había sido instruido de alguna manera sobre las pinturas religiosas que iban a serle útiles para el trabajo de evangelización en Japón. Se sabe por una carta de San

2 Cfr. Fernando $\mathrm{G}^{\mathrm{a}}$ Gutiérrez, S.J.: "Contactos históricos entre Occidente y el Lejano Oriente", Boletín de Bellas Artes, n XVII, Real Academia de Bellas Artes de Santa Isabel de Hungría, Sevilla, 1989.

3 Marco Polo: Il Milione (capit. 155). La Biblioteca di Babele, Clasici della Letteratura Italiana, edición digital [http: //digilander.libero.it/bepi/biblio3a/indice3.html].

$4 \quad$ Para la discusión de esta fecha, Cfr.. la obra de Sennai Kenji: "The European Inloading to the Far East and Japan”, en Outline of Japanese Cultural History, vol. 8, p. 111 (sin datos de lugar y fecha de edición). 
Francisco Javier que Anjiro enseñó a Shimazu Takahisa una pintura de la Virgen, de las que había llevado desde Europa. Dice Javier que el japonés estuvo admirando aquella pintura, y que su madre quiso obtener una copia de ella, pero que no hubo quién la hiciera ${ }^{5}$. Ésta fue la primera introducción de una obra de arte occidental en Japón de que conste en algún documento histórico ${ }^{6}$.

En una relación que escribió Javier sobre la extensión del Cristianismo en Japón, se hace notar que las pinturas religiosas eran un medio muy eficaz para la propagación de la fe entre los japoneses. Esto hizo que la demanda de pinturas religiosas fuera cada vez mayor. Consta históricamente que un gran número de éstas, seguramente en forma de grabados, fue llevado a Japón desde Europa. Entre otras, sabemos también que Javier mostró una Biblia con ilustraciones al señor de Yamaguchi, Ouchi Yoshitaka (1507-1551), y una pintura de la Virgen a Otomo Soria (1530-1587). También sabemos que existió un cuadro de la Virgen para un altar que el jesuita Luis d'Almeida (c.1525-1583) hizo en un hospital de Bungo; unas pinturas para un altar de una iglesia de Yamaguchi; una pintura entregada por el P. Cosme de Torres (15101570) a Omura Sumitada (1533-1587), y un cuadro de la Virgen que fue llevado a Hirado en 1565 y que más tarde cayó en manos del perseguidor del Cristianismo Kato Kiyomasa (1562-1611)7. A pesar de todo, estas obras traídas de Occidente no daban abasto, y se vio pronto la necesidad de formar a pintores japoneses que fueran capaces de reproducir las obras de arte occidental que tenían temas religiosos. La misión de jóvenes japoneses a Roma, que organizó el P. Alessandro Valignano (1539-1606) en 1582, llevó ya algunas obras de temas cristianos hechas por japoneses en estilo occidental. Al volver a Japón llevaron consigo varios objetos de arte occidental que también influyeron en los artistas japoneses, que ya producían obras de arte en estilo de Occidente.

Se fundaron seminarios y colegios en varios sitios de Japón para dar una formación cultural a los alumnos japoneses. En estos centros se enseñaba, además de religión y teología, la música y el arte de Occidente, especialmente la pintura y el arte de grabar. El fin de estos centros era armonizar la cultura cristiana y la japonesa, basados en el sistema educacional de la Compañía de Jesús, que era considerablemente apreciado en Europa en aquel tiempo. El P. Alessandro Valignano (15391606), visitador enviado por el P. General Everardo Mercurian (1573-1580) en 1574 a los jesuitas de Japón, fue quien dio mayor impulso al establecimiento de centros educacionales en aquel país dirigidos por los miembros de la Compañía de Jesús ${ }^{8}$. El mismo Valignano fue quien introdujo la imprenta en estos seminarios, de los que fundó uno en Arima (1580) y otro en Azuchi (1581). En los libros impresos en estos centros aparecieron, en las primeras páginas, unos grabados religiosos hechos en planchas de cobre. Se conservan algunos libros impresos entonces que muestran la maestría alcanzada por los alumnos en el arte de los grabados ${ }^{9}$.

5 Citadio por Miki Tamon: The Influence of Western Culture on Japanese Art. Monumenta Nipponica, Sophia University, Tokyo, 1964, vol. XIX, nº 3-4, p. 164.

6 Cfr. la obra de Ono Tadashige: Yedo no Yodaka, Sansaisha, Tokyo, 1968, p. VI.

Miki Tamon: The Influence of Western Culture on Japanese Art, op. cit., p. 146.

8 Para una descripción detallada de la obra realizada por Alessandro Valignano, S.J. en Japón, Cfr. Fernando G ${ }^{\mathrm{a}}$ Gutiérrez, S.J.: "Valignano and the Introduction of Western Art in Japan, en Alessandro Valignano S.I. Unomo del Rinascimento: Ponte tra Oriente e Occidente". Ponencias del Congreso Internacional tenido en Chieti (Italia), en junio de 2006, y publicadas por el Institutum Historicum Societatis Iesu, Roma, 2008, pp. 175-185.

9 Cfr. Varios de estos libros publicados en los seminarios de los jesuitas en: Arimicho Ebisawa: The Meeting of 
Uno de los misioneros que más influyó en la formación artística de los japoneses fue GIOVANNI COLA ${ }^{10}$. Su nombre es mencionado indistintamente como Nicolao, Nicola o Cola en los Catálogos de la Compañía de Jesús ${ }^{11}$. Nació en Nola (Nápoles) en 1560, e ingresó en la Compañía de Jesús en 1580. Un año más tarde es enviado a Portugal, y en Lisboa embarca para la India. Después de una breve estancia en Goa, sigue hacia Macao, donde en 1583 se encuentra con el P. Alessandro Valignano que pensaba ir a Roma con los cuatro jóvenes Legados de los tres Daimyo cristianos de Kyushu. En Macao pintó Giovanni Cola un cuadro del Salvador (Salvator Mundi), entre agosto de 1582 y julio de 1583. También se encuentra en Macao con el P. Pedro Gómez (1535-1600), que sería más tarde Vice-provincial de Japón, y el P. Francisco Pasio (1553-1612), que también sería después superior en Japón. Valignano se reunió con todos ellos para discutir los métodos de evangelización en Japón, y en ese encuentro tratarían también de los seminarios de arte que se iban a fundar en Japón. Giovanni Cola llegó a Nagasaki en junio de 1583, junto con los PP. Pedro Gómez y Francisco Pasio ${ }^{12}$.

Giovanni Cola era un hombre típico del Renacimiento italiano, con numerosas cualidades artísticas y humanas: era, sobre todo, pintor al óleo, experto en hacer grabados en cobre, matemático, fabricante de complicados relojes y de órganos cuyos tubos estaban hechos con cañas de bambú, etc ${ }^{13}$. Aunque Giovanni Cola no tenía buena salud, pudo unir sus estudios de teología con los de la lengua japonesa, y enseñar en los colegios-seminarios de cultura occidental, que los jesuitas habían establecido en Japón. En los cuatro años que median desde su llegada a Nagasaki y el decreto de destierro dado por Toyotomi Hideyoshi (1536-1598) para todos los misioneros en 1587, su vida se dividió entre Nagasaki, los seminarios de Arima y de otros lugares a donde tuvieron que trasladarse, y la iglesia de Kyoto. Esta última estancia es importante en su carrera artística porque allí entró en contacto con los pintores de la famosa escuela japonesa de Kano de pintura ${ }^{14}$.

Desde 1587 residen los jesuitas en el seminario de Arima, en donde abren su escuela de pintura en 1590. Como el P. Valignano había llevado a Japón la imprenta de caracteres móviles, se comienzan a imprimir libros de catequesis y temas cristianos, que llevan en la primera página grabados en los que trabaja especialmente Giovanni Nicolao. Pero la persecución contra todo lo cristiano arreciaba, y esto hizo que la escuela de arte se tuviera que trasladar a diversos sitios, en que encontraba un poco más de libertad para su actividad: Hachirao, Kazusa, Arie, Arima, Shiki y Nagsaki. Estaba Giovanni Cola en Arie, en 1597, el año del Gran Martirio de los 26 Mártires de Nagasaki, y allí dirigió la ejecución de la pintura y el grabado de Nuestra Señora de la Antigua, conocida en Japón como La Virgen de Sevilla (Fig. 1), que reprodujo

Cultures. The Southern Barbarians. The First Eurpeans in Japan, Kodansha Internacional Ltd., Tokyo, 1971, pp. 125-144.

10 Para una descripción completa de la vida y obra de Giovanni Cola, Cfr. Diego Yuuki, S.J.: "Giovanni Cola, S.J. (Nicolao), el hombre que hizo florecer las piedra", Temas de Estética y Arte, Real Academia de Bellas Artes de Santa Isabel de Hungría, no XVII, Sevilla, 2003.

11 Giovanni Cola aparece en los Catálogos de la Compañía de Jesús en 24 ocasiones, desde el del 20 de diciembre de 1581 (Catalogus Brevis Provinciae Japonicae), hasta el de diciembre de 1623-enero 1624 (De Catalogis Provinciae Japonicae ).

12 Okamoto Yoshitomo: The Namban Art of Japan, Weatherhill/Heibonsha, Tokyo, 1972, p.100.

13 Ebisawa Arimichi: The Meeting of Cultures. The Southern Barbarians. The First Europeans in Japan, op. cit., p. 140 .

14 Diego Yuuki, S.J.: “Giovanni Cola, S.J. (Nicolao). el hombre que hizo florecer las piedras”, op. cit., p. 108. 
un grabado de esta imagen llevado a Japón por los misioneros y que más tarde pasó a Manila, hasta que finalmente fue devuelto a Japón ${ }^{15}$.

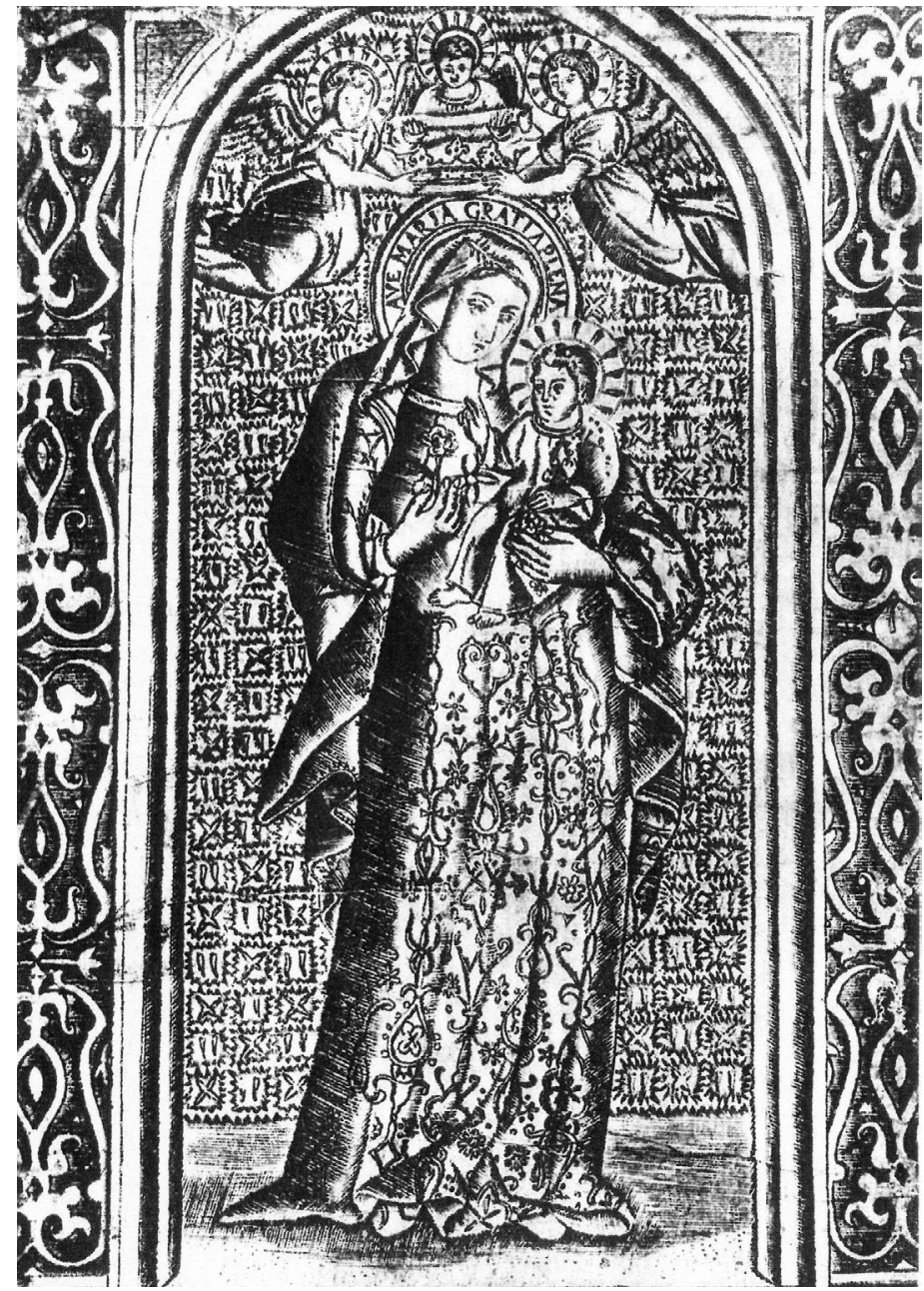

Fig. 1. La Virgen de Sevilla: grabado hecho en 1597, en la Escuela de Arte que los jesuitas tenían en Arie, siguiendo a otro llevado a Japón por los misioneros.

Giovanni Cola pintó varios cuadros del Salvador del mundo, mientras enseñaba en los distintos sitios por los que iba siendo trasladado el seminario de arte. En Nagasaki será donde, desde 1601, realizará su labor más fecunda, en colaboración con varios pintores de la Escuela Kano. Allí se ordenó sacerdote en 1603.

Aunque no se conserva, claramente documentada, ninguna obra de Giovanni Cola, sí hay algunas que pueden atribuirse a él por deducciones circunstanciales. En uno de los Namban-byobu que se conservan en la Colección Imperial de Tokyo, se puede ver claramente, en el interior de una iglesia y puesto sobre el altar, un cuadro

15 Para seguir la historia completa de este grabado, cfr. Diego Yuuki, S.J.: "Giovanni Cola (Nicolao), el hombre que hizo florecer las piedras", op. cit., p. 108, nota 1 . 
con la imagen del Salvador del mundo (Fig. 2), que se sabe era uno de los temas predilectos de Giovanni Cola y que más solía repetir. Tanto, que en alguna carta suya que se conserva, dirigida al P. General de la Compañía de Jesús, P. Claudio Aquaviva (1581-1615), desde Goa en abril de 1581, tiene pìntado junto a su firma, un pequeño rostro del Salvador igual que los que pintaba en sus cuadros. Esto hace pensar que el grabado existente en la primera página del libro Doctrina del Colegio de la Compañía de Jesús, publicado en el Colegio de Amakusa en 1592, fuera hecho por el mismo Cola (Fig. 3).

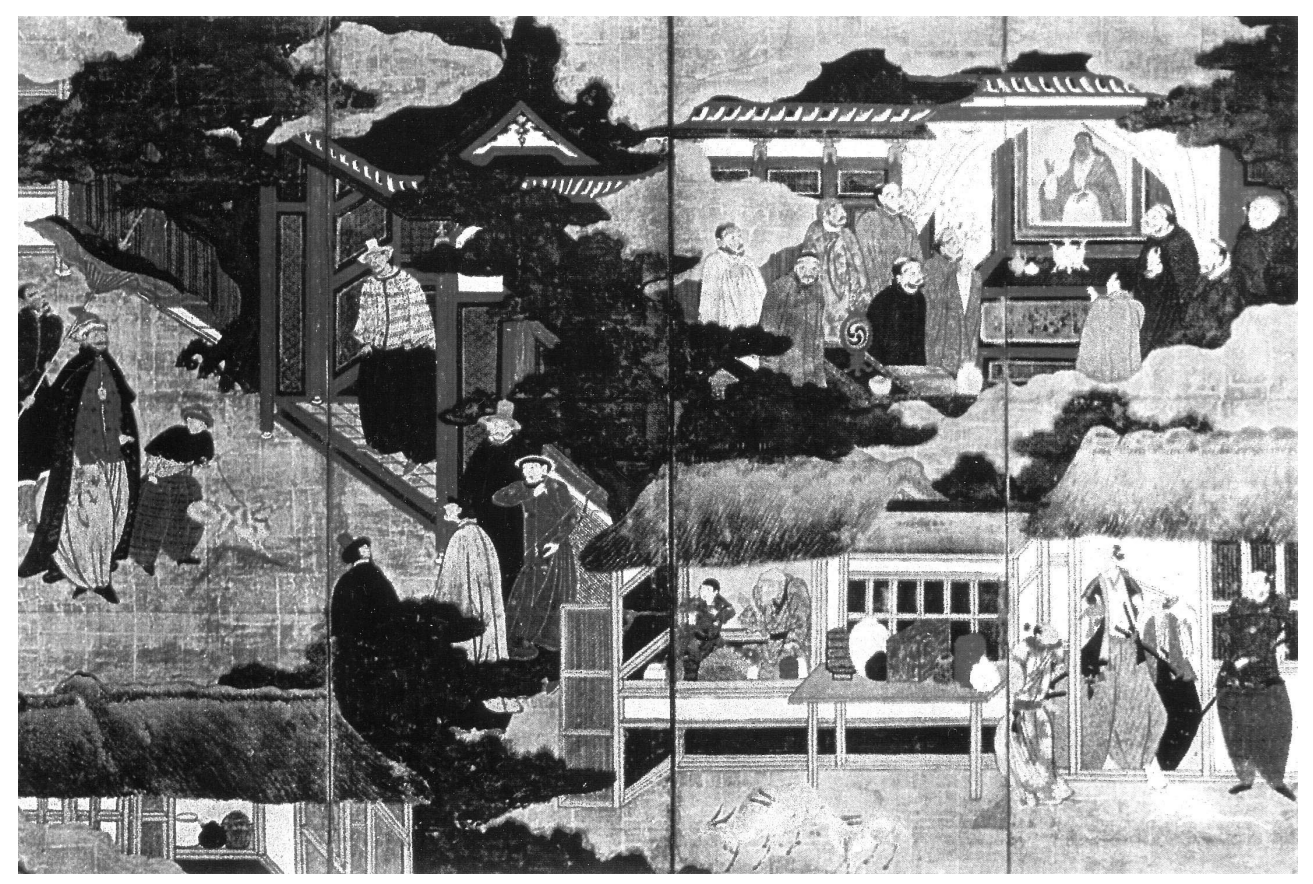

Fig. 2. Biombo Namban, en que aparece sobre el altar la pintura del Salvador del Mundo, que Giovanni Cola pintaba frecuentemente (Colección Imperial, Tokyo).

Se sabe que Giovanni Cola envió a China, ante la petición del P. Mateo Ricci (1552-1610), en 1587, una pintura que representaba al Salvador del mundo, junto con otras que tenían por tema a San Lorenzo y a San Esteban ${ }^{16}$. Un grabado realizado por Johan Wierix del dibujo hecho por Maerten de Vos, tuvo que ser conocido en Japón: se conserva una pintura japonesa, datada en 1597, y que tiene como tema el mismo Salvador del mundo, que es un trasunto del grabado flamenco. Tiene en la parte de atrás escrito en letras latinas Sacam Jacobus. Según algunos especialistas en el arte del Siglo Cristiano en Japón, este nombre es la firma de Jacobo Niwa, un discípulo de Cola, que trabajó más tarde en China. El arte de este discípulo de Gio-

16 Sakamoto Mitsuru: The Rise and Fall of Christian Art and Western Painting in Japan, en la publicación de las ponencias tenidas en el Congreso Internacional The Encounter between Europe and Asia during the Period of the Great Navigations, con motivo del 450 aniversario de la llegada de San Francisco Javier a Japón, Sophia University Press, Tokyo, 1999, p. 127. 
vanni Cola fue muy apreciado por sus contemporáneos ${ }^{17}$, y esta obra de gran calidad artística confirma su importancia como pintor. Ya hemos visto que el tema del Salvador del mundo era uno de los predilectos de Giovanni Cola, que lo transmitió a sus discípulos, como a Jacobo Niwa. Esta es una obra de gran importancia, que sigue al grabado de Johan Wierix, y que Niwa pintó sobre cobre en $1597^{18}$.

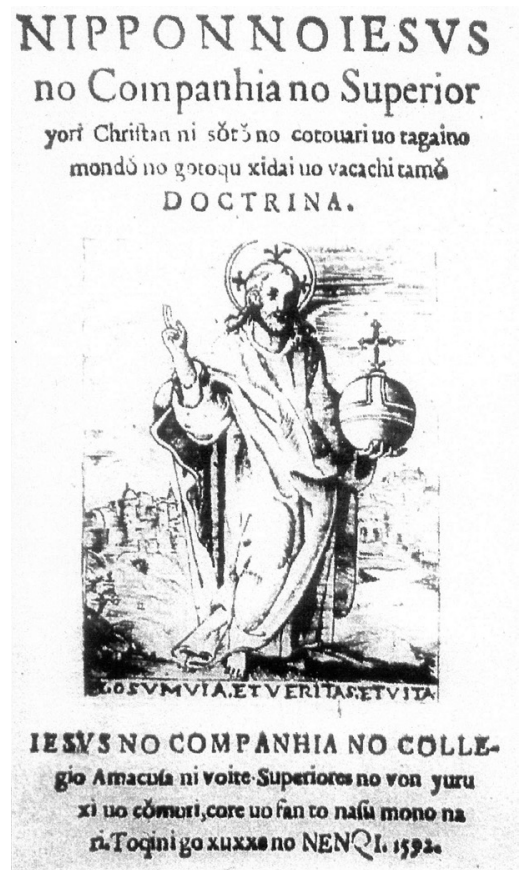

Fig. 3. Primera página del libro de la Doctrina Cristiana, impreso en la Escuela de Arte de los jesuitas en Amakusa, en 1592, con la imagen del Salvador del Mundo.

En 1596 Giovanni Cola cambió de sitio, con el seminario de arte, desde Shiki a Arie, ya que en la Carta Annua de 1596 a Roma se describe la visita del Obispo de Japón, Dom Pedro Martins, S.J. (1591-1598) a aquella academia de arte. En ella se dice que el Obispo y sus acompañantes visitaron el estudio donde se hacían las planchas de cobre para los grabados, el estudio de pintura al óleo, y en todos ellos se quedaron admirados de la habilidad de los alumnos japoneses. Especialmente les llamó la atención una pintura de La Virgen María con el Niño, según el modelo recibido de Italia, en donde es conocida esta pintura como La Virgen de San Lucas ${ }^{19}$.

De Arie pasó Cola a Shiki y Nagasaki, en donde permaneció hasta 1614. Allí siguió enseñando pintura y grabados en cobre, aunque estos últimos sólo los pudo hacer hasta 1611, en que la imprenta fue definitivamente enviada a Filipinas, dadas

17 Cfr. C. R. Boxer: The Christian Century in Japan, University of California Press, Berkeley and Los Angeles, 1967, pp. 199-200.

18 Gustavo Alexander Bailey: The Art of the Jesuit Missions in Japan in the Age of St. Francis Xavier and Alessandro Valignano. En la publicación de las ponencias tenidas en el Congreso Internacional The Encounter between Europe and Asia during the Period of the Great Navigations, con motivo del 450 aniversario de la llegada de San Francisco Javier a Japón, Sophia University Press, Tokyo, 1999, vol. I, p. 199.

19 Citado por John E. McCall: Early Art in the Far East. En Artibus Asiae, vol. X-XII (1947-1949), Dresden, p. 133. 
las dificultades que encontraban los jesuitas ante la persecución de todo lo cristiano. En 1614 se vio obligado a huir a Macao, para escapar de la persecución promovida por el shogun Tokugawa Ieyasu (1542-1616). Allí permaneció, trabajando en la enseñanza del arte occidental, en el Colegio-Universidad de San Pablo, decorando la Iglesia de la Asunción. Murió en 1626, y fue sepultado en aquella iglesia ${ }^{20}$.

Una carta escrita desde Macao por un tal Palmeiro, misionero, describe a Giovanni Nicolao como un auténtico humanista del Renacimiento italiano:

Tenía (Nicolao) una gran elegancia y probidad, con unas cualidades especiales para las matemáticas, la pintura y la fabricación de relojes; estaba dotado con dones especiales para enseñar a sus alumnos la pintura y el arte del grabado; dejó muchas obras pictóricas en las iglesias de Japón y Macao ${ }^{21}$.

En resumen, la figura de Giovanni Cola era la de un verdadero renacentista italiano, que llevó a Japón todo su saber artístico y lo comunicó a sus innumerables alumnos japoneses y chinos en Macao, en una actitud evangelizadora a través del arte.

\section{Pinturas de Giovanni Cola en España}

El Colegio de la Encarnación de la Compañía de Jesús en Marchena (Sevilla) fue de los más importantes que tuvo la orden religiosa en España en el siglo XVI. Fue una institución muy querida por los primeros jesuitas y, debido a la generosidad de los Duques de Arcos sus fundadores, residían en él un buen número de estudiantes jesuitas de Humanidades junto con padres y hermanos. Allí se celebraron los primeras

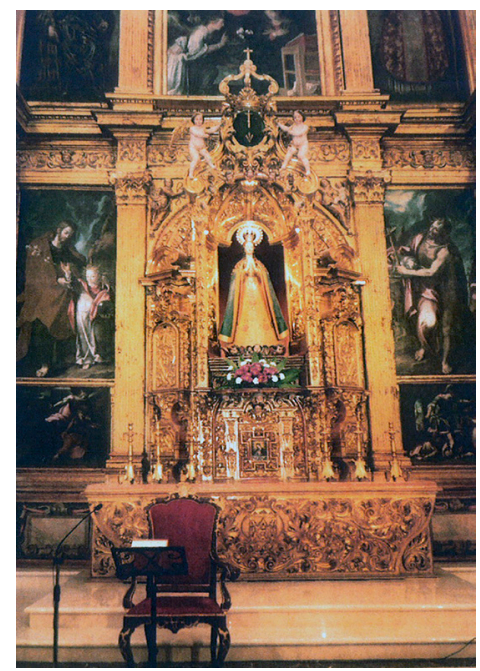

Fig. 4. Altar Mayor de la Iglesia de la Encarnación (actual Iglesia de Santa Isabel), del antiguo Colegio de los Jesuitas en Marchena (Sevilla).

20 Diego Yuuki, S.J. cita el documento del Archivo Ultramarino de Lisboa, Codex 1659, en que se dice que el P. Giovanni Cola murió en Macao el 16 de mayo de 1636, y está enterrado en la iglesia de la Compañía de aquella ciudad: "Giovanni Cola, S.J. (Nicolao), el hombre que hizo florecer las piedras", op. cit., p. 109.

21 Citado por Okamoto Yoshitomo: The Namban Artf Japan, op. cit., pp. 100-101. 
Congregaciones Provinciales de la orden. Poseía este colegio una magnífica iglesia, edificada entre los años 1566 y 1588, con trazas de Hernán Ruiz y del P. Bartolomé de Bustamante (1501-1570)22. El retablo es del $\mathrm{H}^{\mathrm{o}}$ Alonso Matías (1580-1629), realizado en 1610, y en sus calles figuran pinturas de Alonso Vázquez y Juan de Roelas (Fig. 4).

Este Colegio de la Compañía estuvo particularmente vinculado con la misión del Japón, que tenían los jesuitas en aquellas islas desde la llegada de San Francisco Javier en 1549. Hasta tal punto, que se enviaron desde aquella misión las reliquias de los 26 Santos Mártires de Japón, que fueron martirizados el 5 de febrero de 1597, para la iglesia del Colegio de Marchena. Hacia 1970 fueron devueltas estas reliquias a la misión de Japón, para ser colocadas en la capilla del Museo de los 26 Santos Mártires en Nagasaki. Una muestra también de esta vinculación con la misión de Japón es el cuadro existente en esta Iglesia de Marchena, atribuido a Juan de Roelas, en que aparece un gran Crucifijo en la parte central, y San Francisco Javier y San Pablo Miki (uno de los 26 Santos Mártires de Nagasaki) bajo sus brazos ${ }^{23}$ (Fig. 5).

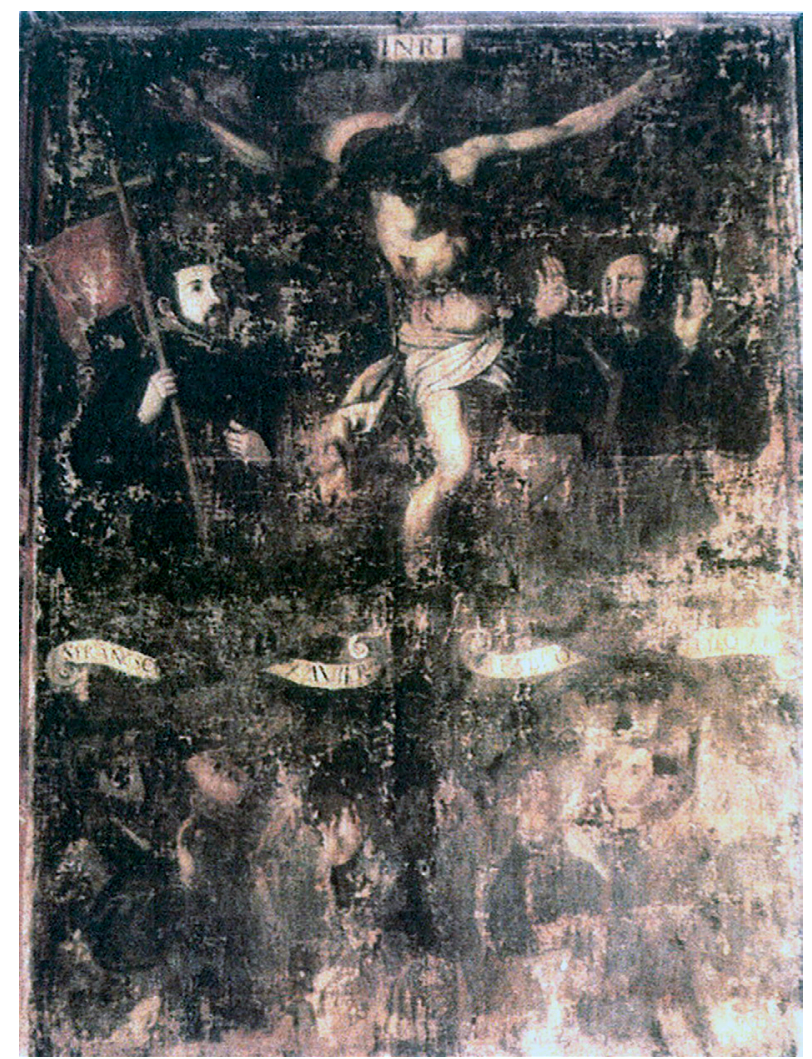

Fig. 5. Juan de Roelas (siglo XVII): Cristo Crucificado con San Francisco Javier y San Pablo Miki. Iglesia de la Encarnación (Marchena, Sevilla).

22 Cfr. Alfonso Rodríguez Gutiérrez de Ceballos, S.J.: Bartolomé de Bustamente y los orígenes de la arquitectura jesuítica en España, Institutum Historicum S.I., Roma, 1967, pp. 211-224.

23 Cfr. Fernando Ga Gutiérrez, S.J.: Los Mártires de Nagasaki. IV Centenario (1597-1997), Ediciones Guadalquivir, Sevilla, 1996, pp. 50-51. 
La comunicación entre el Colegio de la Compañía de Marchena y la Misión de Japón debió ser frecuente. Por eso, no es extraño que la pintura sobre cobre de la Virgen con el Niño (conocida actualmente como La Virgen el Amparo), que está colocada en la puerta del sagrario del altar mayor de la iglesia de este Colegio (Fig. 6), fuera enviada desde Japón por los misioneros. No aparece la mención de esta pintura en las descripciones primitivas del retablo de esta iglesia. Puede haber sido puesta sobre la puerta del sagrario en fecha posterior, como se conserva en la actualidad. Sin duda sería uno de los regalos enviados por los misioneros de Japón para los fundadores de este Colegio, y ellos después lo donarían, como hicieron con otros objetos de arte, para la iglesia del Colegio. Con este envío querrían los misioneros demostrar cómo los alumnos de las academias de arte que tenían en Japón eran capaces de realizar obras tan perfectas en el estilo del arte occidental.

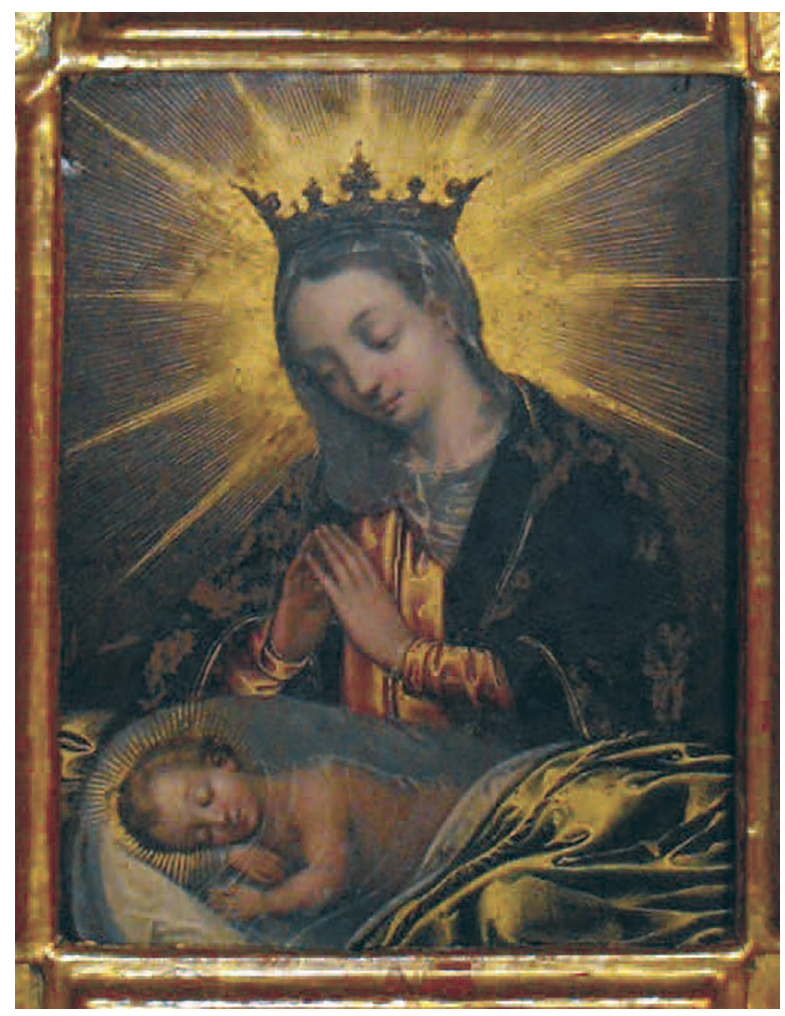

Fig. 6. Puerta del Sagrario del Altar Mayor de la Iglesia de la Encarnación (Marchena), con una pintura realizada en una Escuela de Arte de los jesuitas bajo la dirección de Giovanni Cola.

Se conserva un grabado hecho por Giovanni Cola en la Colección Namban Bunka-kan de Osaka (Japón). Aunque el grabado está realizado en tinta monocroma Fig. 7)., sus líneas generales son prácticamente idénticas a la pintura sobre cobre de la puerta del sagrario de Marchena. De hecho, en los libros que describen a este Colegio se atribuye a Giovanni Cola esta pintura ${ }^{24}$. Si comparamos el grabado de Osaka

24 Manuel Antonio Ramos Suárez: El Colegio de la Encarnación de Marchena, Ed. Codexsa, Sevilla, 2008, p. 51. 
y la pintura de Marchena, encontramos rasgos idénticos: la actitud orante de la Virgen, con las manos juntos, que tiene la vista inclinada hacia el Niño colocado delante de ella; el parecido del rostro, de los vestidos (túnica y manto exterior), en la figura de medio cuerpo de la Virgen; la corona sobre la cabeza de la Virgen; la postura del Niño recostado sobre un cojín, con la cabeza reclinada sobre su brazo derecho y el izquierdo cruzado; el paño que cubre el cuerpo del Niño, etc. La pintura del sagrario de la iglesia de Marchena es un verdadero trasunto del grabado que se conserva en Japón. Por todo esto pensamos que la obra que se encuentra en la iglesia del Colegio de Marchena puede atribuirse al mismo Giovanni Cola, o sería la obra hecha por sus alumnos bajo su dirección en alguno de los seminarios o academias de arte en que daba clases de pintura. No olvidemos que Giovanni Cola fue el introductor de la pintura al óleo de Occidente en Japón.

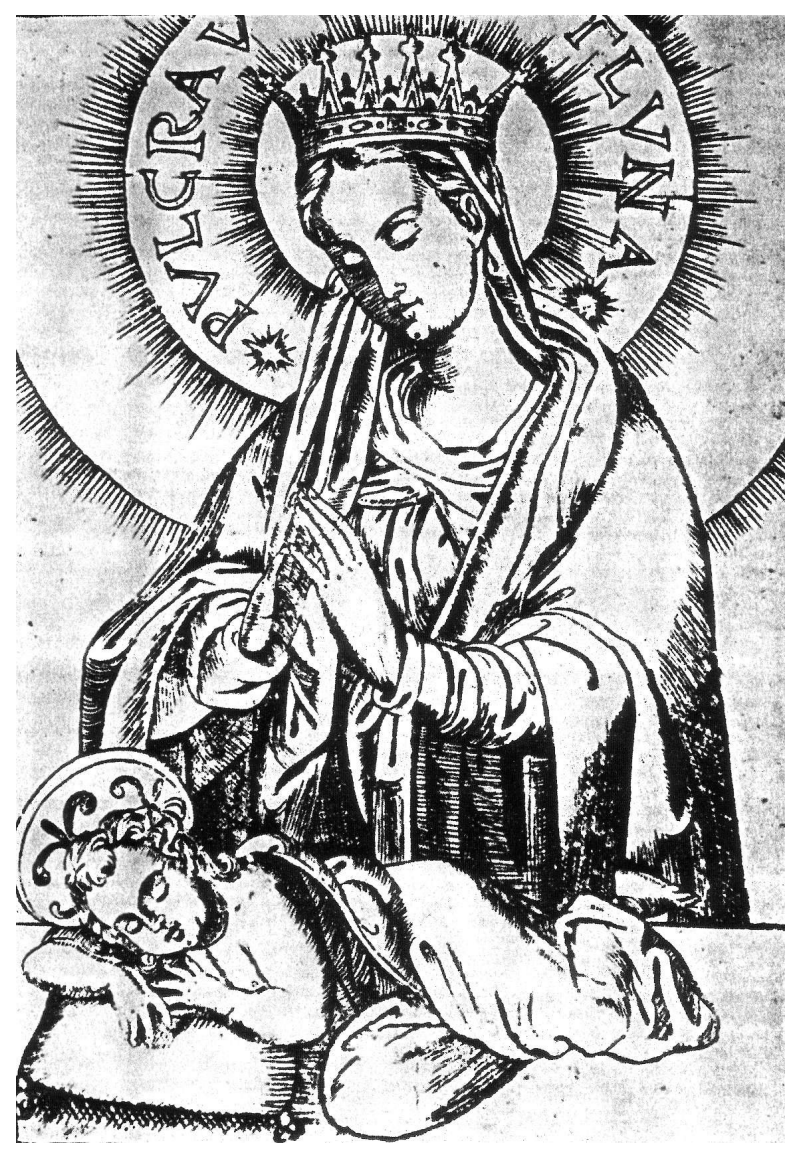

Fig. 7. La Virgen con el Niño: pintura de Giovanni Cola que se conserva en el Museo Namban Bunkakan, de Osaka.

Ha sido recientemente descubierta una pintura, atribuible a Giovanni Cola, que fue donada al Museo de los 26 Mártires de Nagasaki: La Virgen de las nieves (Yuki no Santa Maria) (Fig. 8). Según el Prof. Gaivin Alexander Bailey, esta pintura fue realizada después de 1583 en el taller de Giovanni Cola, y es una de las pinturas más 
bellas de las que se conservan de todas las misiones de los jesuitas ${ }^{25}$. Es una pintura realizada sobre papel, en forma de kakemono (pintura colgante), con pigmentos japoneses de color. La actitud adorante de la Virgen, con la cabeza inclinada, los ojos bajos, el arreglo del pelo y las manos juntas, es la misma que la de la pintura de la puerta del tabernáculo de Marchena. Los mismos tonos empleados en la túnica y el manto son también idénticos en ambas obras. Una misma mano aparece en estas pinturas, que indican el modelo marcado por Giovanni Cola en sus pinturas de las Madonnas. Esta pintura de La Virgen de las Nieves es, además, un hito en el camino de la enculturación del arte occidental en Japón, ya que une las características de las Madonnas italianas a la tradición más típicamente japonesa. Sólo un genio como Giovanni Cola podría haber realizado una obra así.

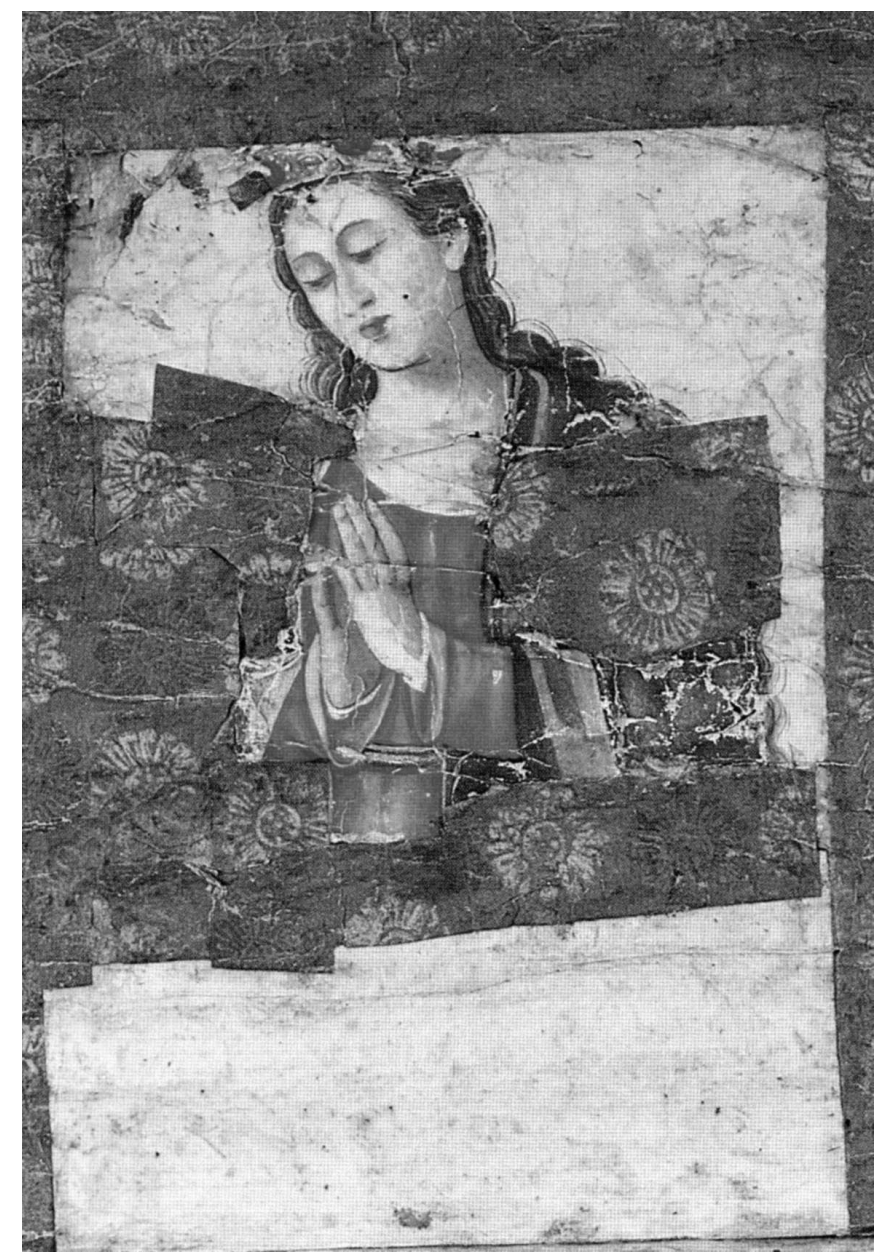

Fig. 8. La Virgen de las Nieves: pintura realizada en alguna de las escuelas de los jesuitas bajo la dirección de Diovanni Cola, que se conserva en el Museo de los 26 Santos Mártires de Nagasaki.

25 Gauvin Alexander Bailey: The Art of the Jesuit Missions in Japan in the age of St. Francis Xavier and Alessandro Valignano, op. cit., vol. I, p. 200. 
Existen también otras pinturas, parecidas a la de la Iglesia de la Encarnación (actual Iglesia de Santa Isabel), en otros lugares de Marchena. La más parecida es la que también está colocada en la puerta del sagrario del altar mayor del Convento de la Inmaculada Concepción, de las RR. Clarisas (Fig. 9). La pintura, también sobre cobre, es casi idéntica a la del sagrario de la Iglesia de la Encarnación, aunque varían algunos detalles. La Virgen está también en actitud orante (Fig. 10), con las manos juntas, los ojos bajos mirando al Niño que está dormido delante de ella, con los mismos colores en su túnica y manto; la postura del Niño también es la misma: dormido con la cabeza sobre el brazo derecho, y una especie de tul transparente que le cubre la parte superior del cuerpo desnudo y cubierto con una tela que le tapa desde la cintura para abajo. La Virgen no tiene la corona, sino sólo un resplandor que emana desde la cabeza. No cabe duda que el modelo es el mismo que veíamos en el grabado de Giovanni Cola, que se conserva en el Museo Namban Bunkakan, de Osaka (Japón). El Niño, colocado delante de la figura orante de la Virgen, está situado más bajo que en la pintura de la Iglesia de la Encarnación. Pero los gestos, el colorido, etc. son idénticos en las dos obras.

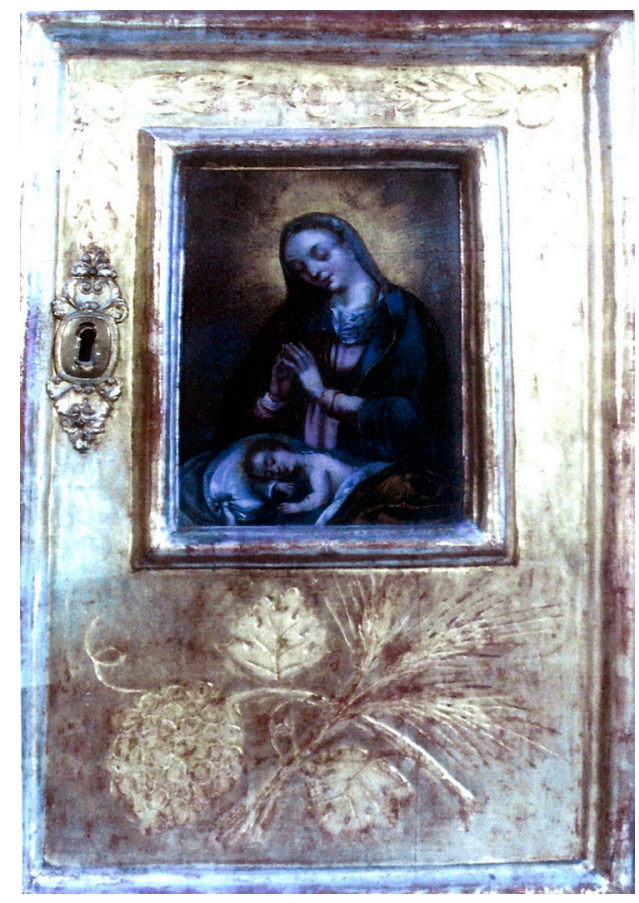

Fig. 9. La Virgen con el Niño: Puerta del Sagrario con una pintura realizada en alguna de las escuelas de arte de los jesuitas en Japón, bajo la dirección de Giovanni Cola (Iglesia del Convento de las RR Clarisas (Marchena, Sevilla).

No cabe duda que el modelo de las dos obras es el mismo. Seguramente las dos fueron enviadas desde Japón por los misioneros que habían establecido aquellos colegios de arte como medio de evangelización; en ellos había talleres de pintura religiosa, y los alumnos japoneses de Giovanni Cola eran los que pintaron estas obras bajo su dirección. En general, la calidad artística de la pintura de la Iglesia de la Encarnación es más alta, pero las dos tienen un mismo modelo inspirador. Serían 
enviadas por los misioneros a sus bienhechores de Marchena como un signo de agradecimiento, y para mostrar la capacidad de asimilación que se había logrado en los alumnos japoneses del arte cristiano de Europa.

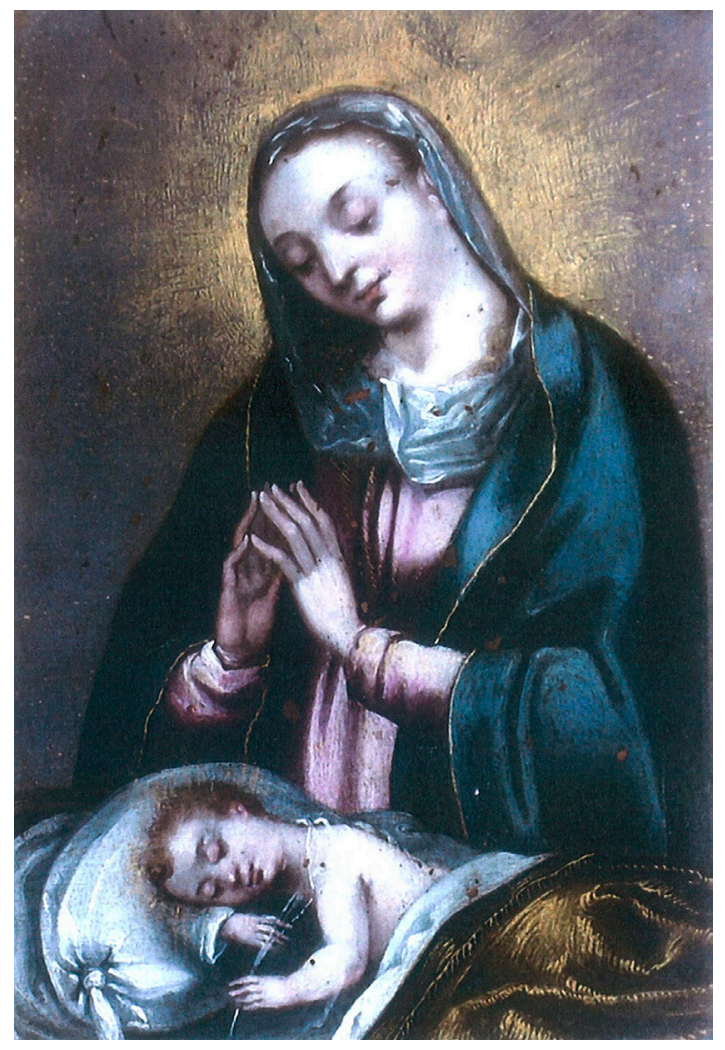

Fig. 10. La Virgen con el Niño: Pintura en la puerta del Sagrario de la Iglesia de las RR. Clarisas (Marchena).

Es interesante observar que en el British Museum de Londres existen tres grabados que representan a la Virgen en adoración del Niño que tiene delante, y que muestran un parecido indiscutible con las pinturas del taller de Giovanni Cola que hemos descrito. Dos de ellas presentan a la Virgen en la misma postura de adoración, con el Niño acostado delante de ella, igual que en las pinturas del taller de Giovanni Cola que están en Marchena. Los dos grabados están firmados por Hieronymus Wierix, de la gran Escuela Flamenca de grabadores ${ }^{26}$. En el primero de éstos (Fig. 11) está la Virgen vuelta hacia el lado derecho y en el segunda está vuelta hacia el lado izquierdo (Fig. 12).

Parece casi cierto que estos grabados debieron ser vistos por Giovanni Cola, y que de ellos recibiera la inspiración para las pinturas que realizó más tarde en Japón, e incluso que llevara algunos apuntes de estos grabados cuando los conociera. Esto se confirma al ver un tercer grabado (Fig. 13), también existente en el British

26 Los dos grabados pertenecen al Departamento de Grabados del British Museum, de Londres, y están registrados con los números 1859,0709.3030 y 1859,0709.3031. 


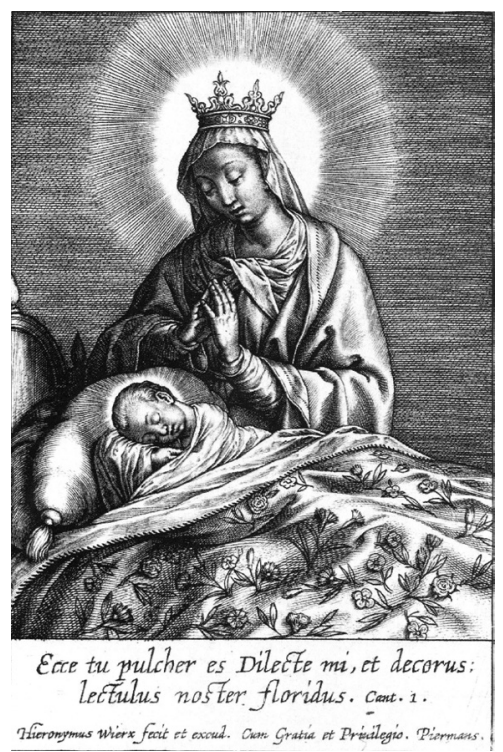

Fig. 11. Hiernonymus Wierix: La Virgen con el Niño. Grabado, 1619 (British Museum, Londres).

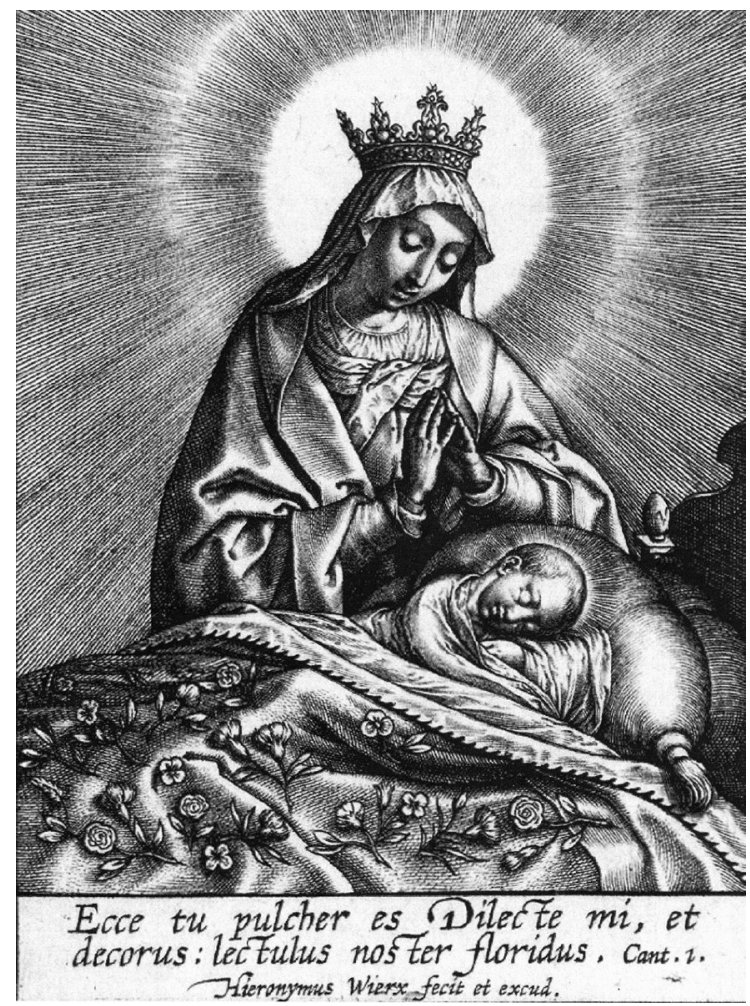

Fig. 12. Hiernonymus Wierix: La Virgen con el Niño. Grabado, 1619 (British Museum, Londres). 
Museum, que no tiene la firma de Hieronymus Wierix y que en la ficha del Museo se atribuye a un Seguidor de Hieronymus Wierix ${ }^{27}$. Muy probablemente este grabado pudo ser realizado por el mismo Giovanni Cola, después de ver los de Hieronymus Wierix, ya que la semejanza con las pinturas de Marchena es mucho mayor. Los datos descriptivos de la Virgen y el Niño son algo distintos de los dos grabados anteriores, aunque hay también muchas semejanzas en las figuras de la Virgen y del Niño. Sin duda Giovanni Cola fue un investigador asiduo de los grandes grabadores de la Escuela Flamenca del siglo XVI, y pudo realizar este grabado antes de su marcha a Japón. No es extraño que después repitiera en las pinturas los grabados que había realizado antes en Europa.

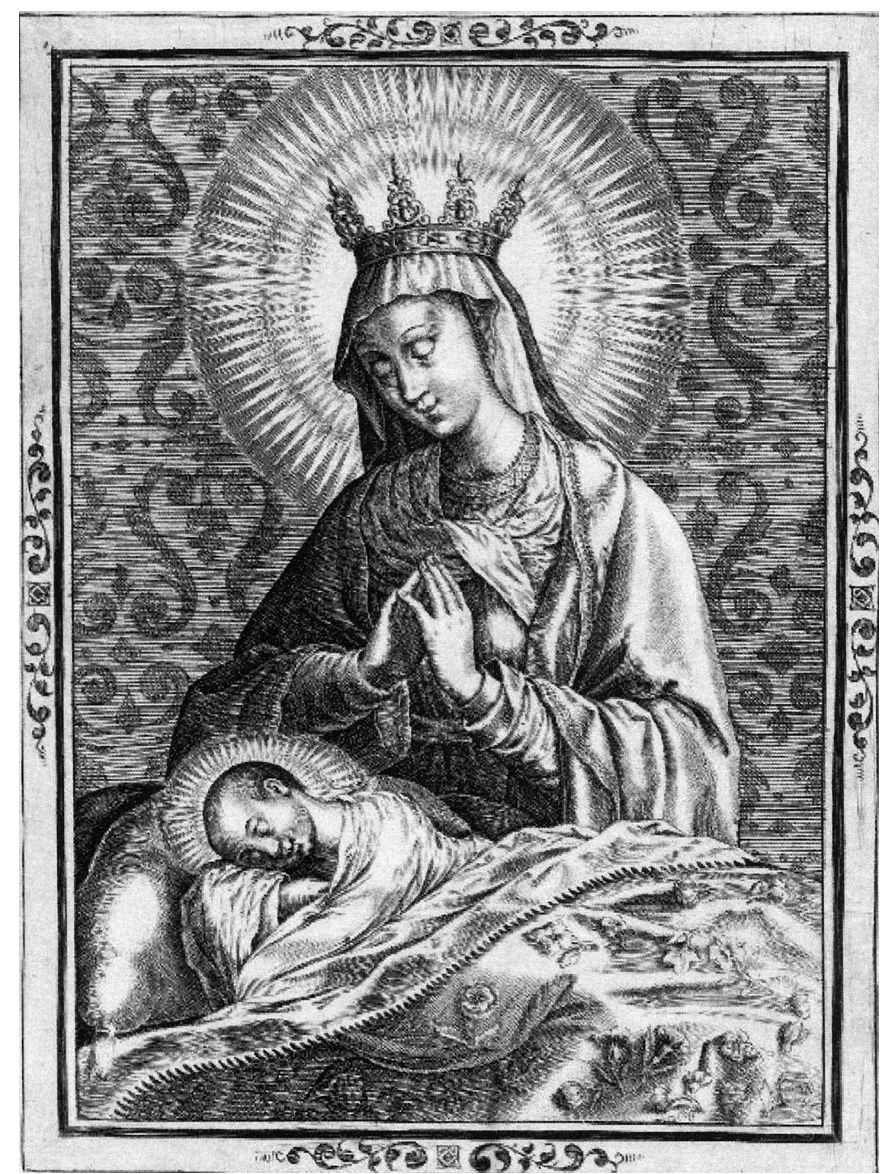

Fig. 13. Giovanni Cola (Atribuido): La Virgen con el Niño. Grabado, 1619 (British Museum, Londres).

Es interesante el poder admirar estas pinturas debidas a la inspiración de Giovanni Cola en España, concretamente en Marchena (Sevilla). Como ya indicábamos, se da en ellas esa unión de los datos estéticos de la pintura italiana con los de la japo-

27 La referencia de este tercer grabado en la ficha del British Museum de Londres es: 1874,0711.1986, también en el Departamento de Grabados de este Museo. 
nesa, en un alarde de enculturación de los temas cristianos en la pintura de Japón. Hasta la llegada de Giovanni Cola a aquellas islas, el arte de Europa era muy poco conocido, y fue él quien introdujo y enseñó por primera vez a los japoneses la técnica occidental de la pintura al óleo en las academias establecidas allí por los jesuitas.

Este artículo fue publicado en Temas de Estética y Arte. Academia de Bellas Arte Santa Isabel de Hungría, ${ }^{\circ}$ XXV (Sevilla:, 2011, pp. 99-123). Por la gentileza de la Academia de Bellas Arte Santa Isabel de Hungría, se reedita aquí a modo de homenaje póstumo al P. Fernando García Gutiérrez (Jerez de la Frontera, 1928-Málaga, 2018), miembro de honor de la Asociación de Estudios Japoneses. Nuestro agradecimiento a la Academia. de Bellas Artes de Sevilla, de la cual el P. Fernando García Gutiérrez fue activo miembro académico. Lo publicamos aquí respetando su primera edición. 